\title{
Transient Stability of Surfactant and Solid Stabilized Water-in-Oil Emulsions
}

D. Venkataramani ${ }^{1,2}$, J. E. Smay ${ }^{3}$, C. P. Aichele ${ }^{1,2 *}$

${ }^{1}$ School of Chemical Engineering, Oklahoma State University, Stillwater, OK 74078, United States

${ }^{2}$ Center for Interfacial Reaction Engineering (CIRE), School of Chemical Engineering, Oklahoma State University, Stillwater, OK 74078, United States

${ }^{3}$ School of Materials Science and Engineering, Oklahoma State University, Tulsa, OK 74106, United States

\section{ABSTRACT}

Water-in-oil emulsions with a dispersed phase fraction equal to $15 \mathrm{wt}$ \% were prepared using either a non-ionic surfactant or solid particles under identical conditions of high energy mixing. Acoustic spectroscopy measurements were carried out on these emulsions to determine the initial droplet size distributions and their evolution over a period of one week. A transient stability test was conducted to compare the behavior of surfactant and solid particles at the oilwater interface. The objective of this paper was to elucidate the use of acoustic spectroscopy for characterizing emulsion stabilization/destabilization mechanisms in these emulsions. Experimental results indicate that a destabilization mechanism, such as coalescence, can be characterized using acoustic spectroscopy. Characteristics prevalent in the coalescing system, such as broad droplet size distribution and polydispersity, were captured by acoustic spectroscopy in terms of change in the raw experimental attenuation. Complimentary bottle test and optical microscopy experiments were conducted on these emulsions to validate the droplet size distribution and transient behavior of emulsions measured using acoustic spectroscopy.

Keywords:

Solid stabilized emulsions (pickering emulsions), acoustic spectroscopy, droplet size distribution, transient stability, conductivity, colloidal vibration current 


\section{Introduction}

Emulsions are metastable mixtures of two or more immiscible liquids. They can be classified as oil-in-water or water-in-oil based on the nature of the dispersed and continuous phase. An emulsion is called stable if there is no phase separation against any destabilization phenomenon, such as coagulation and coalescence, phase inversion, creaming, sedimentation and Ostwald ripening, and if the emulsification process is possible [1]. Emulsifiers are additives that stabilize the oil-water interface. Surfactants are the usual choice for this purpose, and have been available in the market as an emulsifier for both water-in-oil and oil-in-water emulsions. Surfactants are either ionic, non-ionic or zwitterionic in nature. Surfactants reduce interfacial tension, thereby promoting the creation of water-oil interface, and subsequently stabilize the interface to resist coalescence [2, 3].

Emulsions stabilized using finely divided particles/ nanoparticles are called solid stabilized emulsions (also known as Pickering emulsions) [2, 3]. Solid stabilized emulsions have gained importance in the last two decades due to their applicability in many industries such as food, agriculture, pharmaceutical etc., enhanced emulsion stability, and ability to produce droplets ranging from a few microns to several millimeters [4]. Pickering identified that solid particles had the ability to produce stable emulsions, and therefore, these are called Pickering emulsions [2, 3]. Solid particles function in a manner similar to surfactants. However, the preferential wetting of solid particles plays a major role in determining whether oil-in-water or water-in-oil emulsions are formed [5]. Solid particles such as pre-treated silica (available in the market as Aerosil) have been widely used in the pharmaceutical and cosmetic industries as a stabilizer, and have paved a way into the oil and gas industry for producing stable and large droplet emulsions [6]. Solid particles such as hydrophobic silica are known to be preferentially 
wetted by the oil phase, and lead to the formation of water-in-oil emulsions; whereas, hydrophilic silica particles, which are preferentially wetted by the water phase, produce stable oil-in-water emulsions $[3,7,8]$.

Solid stabilized emulsions can be more stable, depending on surface coverage, as compared to surfactant stabilized emulsions, due to the strong adhesion of solid particles to the oil-water interface. In solid stabilized emulsions, the most important factors to consider are the contact angle, coalescence kinetics, and particle interaction energy [7]. Stable emulsions are prepared when the particles are neither too hydrophilic nor hydrophobic and if there is complete surface coverage. Particles that form contact angle, $\theta>90^{\circ}$ form a water-in-oil emulsion, whereas, $\theta<90^{\circ}$ leads to an oil-in-water emulsion $[5,7,8]$. The particle shape, size and concentration greatly affect the emulsion stability and droplet size [9].

Acoustic and electroacoustic spectroscopy are some of the most reliable techniques for characterizing concentrated dispersions and emulsions in-situ [10]. These ultrasound based methods were chosen over other traditional particle sizing techniques, such as dynamic light scattering, electron microscopy, etc., due to their ability to characterize polydisperse and concentrated systems without dilution [10, 11]. For this study, we used an Acoustic/Electroacoustic spectrometer DT 1202 (produced by Dispersion Technology Inc.) for characterizing droplet size distribution of both surfactant and solid stabilized emulsions [10, 11].

The motivation behind this work was to demonstrate how acoustic spectroscopy can be utilized to capture emulsion stabilization/ destabilization phenomena in surfactant and solid stabilized emulsions, and characterize their transient stability over a period of time. For the purpose of this work, emulsions that exhibit two different kinds of stabilization/ destabilization mechanisms were studied using acoustic spectroscopy: one that undergoes coalescence, and a 
second that exhibits high stability. A surfactant stabilized emulsion was used for investigating the transient behavior of a highly stable emulsion, since it is well known to produce stable and non-coalescing droplets when formed above the critical micelle concentration. Since coalescence in surfactant stabilized systems is well studied and understood, a solid stabilized emulsion was chosen for studying acoustic interrogation of a coalescing system. Another important goal of this work was to investigate how acoustic spectroscopy captures characteristics prevalent in coalescing droplets, such as polydispersity and broad drop size distribution. For the purpose of this study, both coalescing and highly stable emulsions were prepared using similar mixing conditions and composition.

In this paper, the droplet size distribution of water-in-oil emulsions were stabilized using hydrophobic fumed silica (aerosil R972) and a non-ionic surfactant, sorbitan monooleate (span80), and were studied at different time intervals after emulsification. Bottle test and optical microscopy measurements were also carried out to validate the results obtained from acoustic spectroscopy.

\section{Materials and Methods}

\subsection{Emulsion Preparation}

The water-in-oil emulsions prepared for this study contained decalin as the oil phase and deionized water as the aqueous/ dispersed phase. Decalin (purchased from BDH chemicals) with $99 \%$ purity and a density of $0.786 \mathrm{~g} / \mathrm{cm}^{3}$, and deionized water with a resistivity of $18.2 \mathrm{M} \Omega \mathrm{cm}$ were used. Sorbitan monooleate (span 80) and hydrophobic fumed silica (Aerosil R972) were the two different stabilizers used for this study. 
For the surfactant stabilized emulsion, span $80, \mathrm{C}_{24} \mathrm{H}_{44} \mathrm{O}_{6}$ (molecular weight $=428.6$ $\mathrm{g} / \mathrm{mol}$ ), supplied by Sigma Aldrich with a density of $0.99 \mathrm{~g} / \mathrm{cm}^{3}$, was used as an emulsifier. The critical micelle concentration of span 80 in solvents such as decalin (dielectric constant $=2.2$ ) was identified as 1.5-2 wt\%[12]. Hence, the oil phase was composed of $2 \mathrm{wt} \%$ of span 80. Span 80 is used in industries as a surface-active emulsifier. Span 80 is a non-ionic lipophilic emulsifier with a hydrophile-lipohile balance ratio (HLB) of 4.3. The low HLB ratio of Span 80 is well known to produce w/o emulsion.

For Pickering emulsions, hydrophobic fumed silica (Aerosil R972) manufactured by Evonik Industries (Rheinfelden, Germany), with a tamped density of $1.06 \mathrm{~g} / \mathrm{cm}^{3}$ was used as the stabilizing agent. Aerosil R972 is manufactured by flame pyrolysis of silica at $1000{ }^{\circ} \mathrm{C}$ and surface treated with dichlorodimethyl silane to impart hydrophobic functionality. The primary particle size of Aerosil R972 is $16 \mathrm{~nm}$, and the material typically exists as a sintered aggregate with size varying in the size range $100-300 \mathrm{~nm}$. To keep the emulsifier concentration constant for both kinds of emulsions, 2 wt\% aerosil R972 was dispersed in the oil phase.

Both types of emulsions were prepared using an Ultraturrax T25 homogenizer with a maximum operating power of $25,000 \mathrm{rpm}$. The oil phase of the emulsions was prepared by mixing 81.98 vol.\% of Decalin with 2.12 vol.\% of the stabilizer using a spatula, and homogenizing at $8000 \mathrm{rpm}$ for $1 \mathrm{~min}$ to completely disperse the stabilizer in the oil phase. 15.9 vol.\% of water was slowly added to the oil phase, and the emulsion was prepared using the homogenizer at $8000 \mathrm{rpm}$ for 10 minutes. 


\subsection{Acoustic Spectroscopy}

For this paper, we used an acoustic/electroacoustic spectrometer DT-1202 (manufactured by Dispersion Technology, Inc.) for characterizing both kinds of emulsions. A detailed description of this device is available in the literature [13]. The spectrometer is used for measuring drop size distribution, zeta potential, and conductivity simultaneously. The acoustic technique utilizes acoustic sound waves for measuring particle and drop size distributions; whereas, the electroacoustic technique utilizes both electric and acoustic signals for measuring surface charge and zeta potential.

Acoustic particle sizing is regulated by two International Standards: ISO 20998 Parts 1 and $2[10,11,13]$. The two essential features of the acoustic particle sizing technique that make it absolute and not requiring calibration are pulse technique and variable gap method. The acoustic spectrometer works on the principle of generating pulses at 18 different frequencies within the range from 1 to $100 \mathrm{MHz}$, and 21 gaps between transmitter and receiver from $0.3 \mathrm{~mm}$ to $20 \mathrm{~mm}$. A piezoelectric transducer (transmitter) converts these signals to ultrasound pulses of the same frequency. These ultrasound pulses propagate through a liquid sample, interact with the liquid and particles, and consequently attenuate. A second transducer (receiver) converts the received sound pulse into electric signals. The difference in the initial and final electric pulses serves as a measurement of energy loss within the sample and is measured as attenuation. This energy loss is measured at variable gaps and frequencies between the transmitter and receiver. The variable gap method is coupled with the Beer-Lambert law for calculating attenuation coefficient, $\alpha$, and per unit length $(\mathrm{dB} / \mathrm{cm} / \mathrm{MHz})$ [14]. Like density, viscosity, and sound speed, acoustic attenuation is an intrinsic property, and unique to each system. The acoustic spectrometer measures the raw experimental attenuation, which is then fit into theoretical models 
to predict the drop size distribution. The theoretical model used to predict the drop size distribution is a function of the experimental attenuation and intrinsic properties of the phases of the system. The software searches for the drop size distribution that provides the best fit to the experimental attenuation spectra, and predicts the mean droplet size and distribution.

\subsection{Optical Microscopy}

For this work, a visualization technique was employed to quantify droplet size distributions and transient emulsion behavior through the use of an Olympus BX53 polarized optical microscope with shear cell and temperature control $\left(-50^{\circ} \mathrm{C}\right.$ to $\left.450^{\circ} \mathrm{C}\right)$ stage.

\subsection{Experimental Procedure}

An emulsion sample of $180 \mathrm{~g}$ was prepared as described in the emulsion preparation method. Immediately after emulsification, the sample chamber of DT 1202 was filled with the emulsion, and the particle size was measured and reported in Table 1 as " 0 th $h o u r$ ". Transient emulsion stability and evolution of droplet size was studied at different time intervals. Measurements were reported as " $48^{\text {th }}$ hour" and "after 1 week" of emulsification. During the entire process, the emulsion samples were constantly stirred on a Thermo Scientific Inc. (Ashville) magnetic stir plate with a $25.4 \mathrm{~mm}$ long stir bar at $800 \mathrm{rpm}$. The DT 1202 spectrometer was set up with a peristaltic pump operating at half of the full speed range to ensure that there was no phase separation within the sample chamber.

Bottle test experiments were conducted to study the emulsion behavior and any deemulsification phenomena that might occur in these emulsions at different time intervals. Bottle tests simply consist of placing a known amount of sample into a bottle and subsequently 
observing the behavior of the sample as a function of time. Optical microscopy was also employed to measure drop size distributions.

\section{Results and Discussion}

\subsection{Emulsion stability using bottle tests}

The stability of emulsions against coalescence and phase separation was monitored and assessed using conventional bottle test methods. Sedimentation of the emulsion and separation of the oil phase was observed in both surfactant and solid particle stabilized emulsions (Figure 1). However, separation of the oil phase in the solid stabilized emulsions was predominant at alltime intervals when compared to surfactant stabilized emulsions. As seen in Figure 1, though sedimentation in surfactant stabilized emulsion is present, the cloudiness seen in the supernatant likely indicates a large amount of stable, small droplets being kept in suspension by Brownian motion. Rapid separation of the oil phase and sedimentation of droplets seen in solid stabilized emulsions indicate two likely possibilities: a) the droplets are coalescing due to insufficient surface coverage, or b) droplets are experiencing sedimentation due to a density difference, with or without any change in droplet size. No aqueous phase separation was noticed in either type of emulsion even after 1 week of emulsification.

The evolution of both emulsions over time was followed simultaneously using optical microscopy and acoustic spectroscopy for up to 1 week after emulsification. The results obtained from these techniques are discussed in the following sections. 

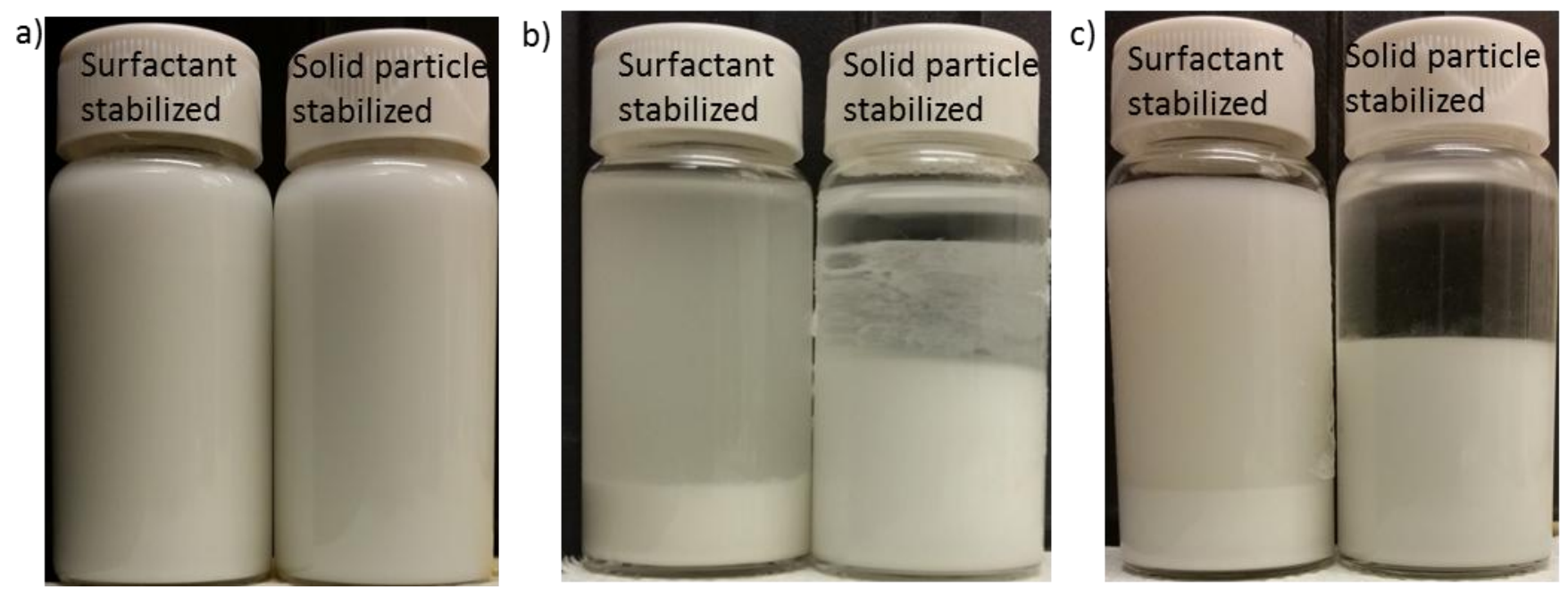

Figure 1: Bottle test experiment showing the stability, phase separation and sedimentation of water droplets in surfactant stabilized water-in-oil and solid particle stabilized water-in-oil emulsion at different time intervals of sample preparation: a) $0^{\text {th }}$ hour, b) after 48 hours of emulsion preparation, c) after 1 week of emulsion preparation. The sample was not stirred throughout this experiment. 


\subsection{Emulsion characterization: optical microscopy and acoustic/electroacoustic}

spectroscopy
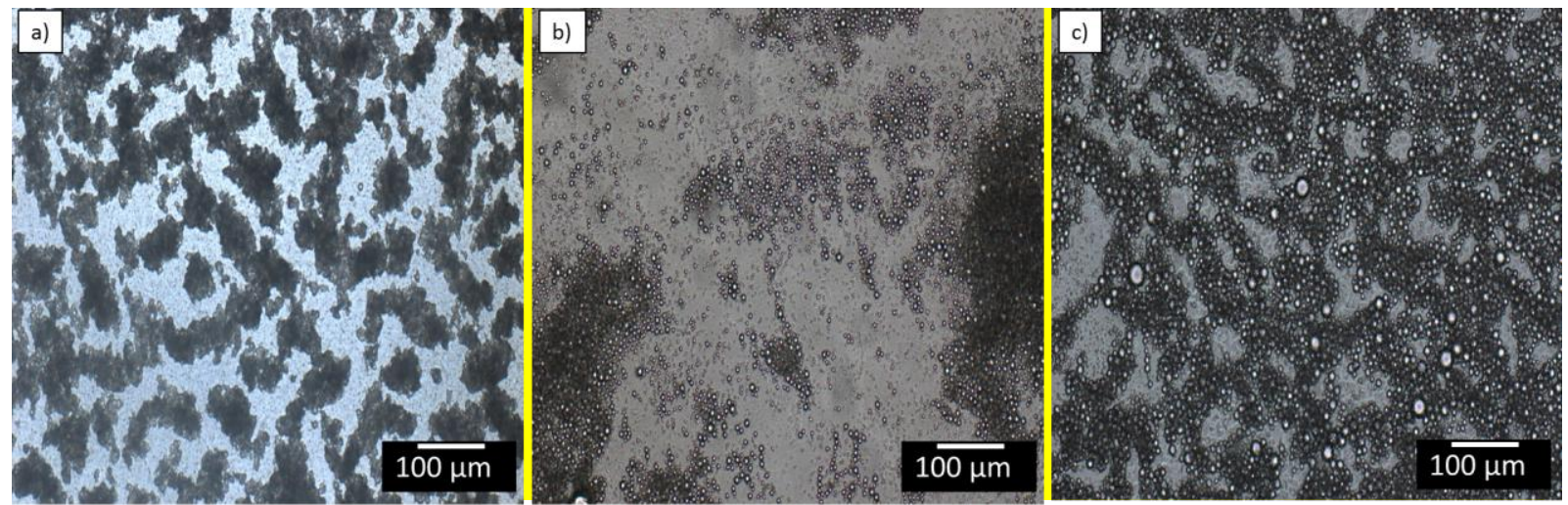

Figure 2: Optical microscopy images (20X magnification) of surfactant stabilized water-indecalin emulsion taken at different time intervals after emulsification a) $0^{\text {th }}$ hour b) $48^{\text {th }}$ hour c) after 1 week.
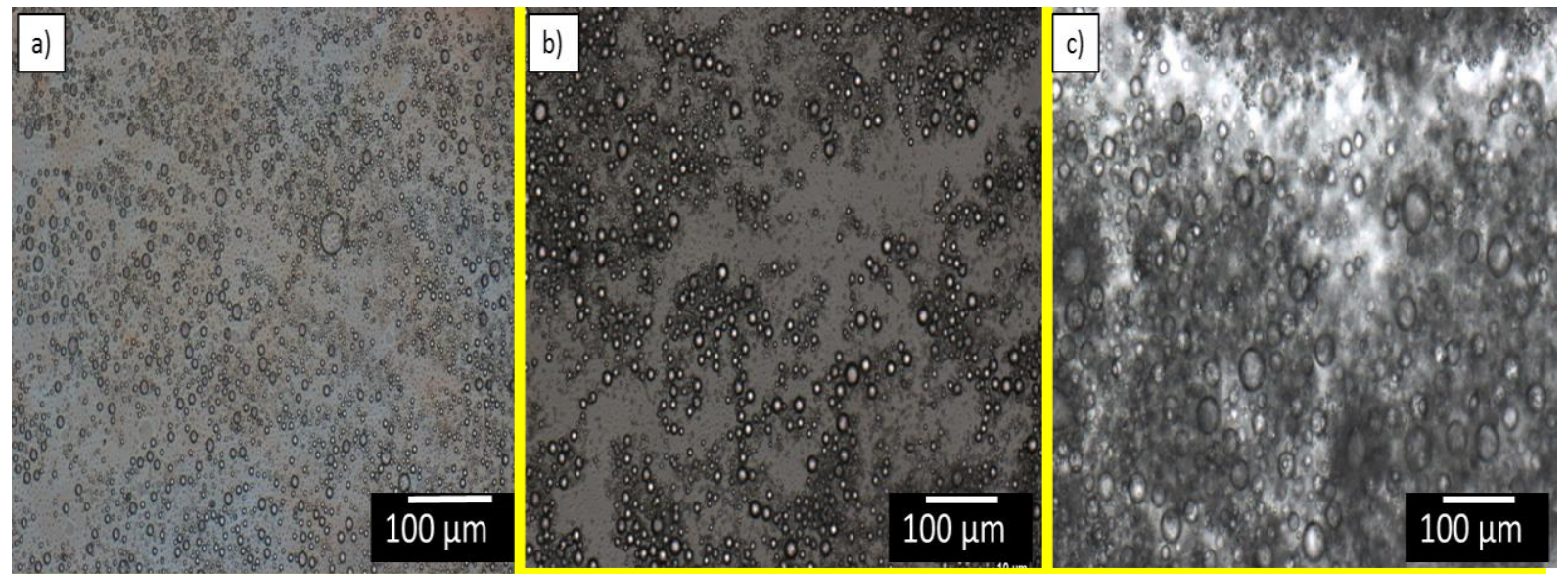

Figure 3: Optical microscopy images (20X magnification) of solid stabilized stabilized water-indecalin emulsion taken at different time intervals after emulsification a) $0^{\text {th }}$ hour b) $48^{\text {th }}$ hour c) after 1 week.

Optical microscopy experiments were conducted on the emulsion samples at time intervals at which bottle test experiments were carried out. Images shown in Figure 2 a) indicate 
that the droplet size distribution centered around $2 \mu \mathrm{m}$ for the span 80 stabilized emulsion at the $0^{\text {th }}$ hour measurement. Whereas, in the solid stabilized emulsion, large droplet size distribution with a mean size centered around $8 \mu \mathrm{m}$ was observed (Figure 3 a). The solid stabilized emulsion also reveals polydisperse character with droplets in the size range of 2- $20 \mu \mathrm{m}$. Images shown in Figure 2 indicate that for span 80 stabilized emulsions, changes in the morphology of the droplets were not observed with time. There was only a slight shift in the mean droplet size from 1.5 to 3 $\mu \mathrm{m}$ after 1 week of emulsification. Drelich et al., observed similar behavior in water-in-paraffin oil emulsion stabilized using span80 [1]. The authors observed that up to 7 days after emulsification, there was only a slight shift in the droplet size distribution from 1.5-3 $\mu \mathrm{m}$ [1].

Images in Figure 3 indicate the evolution of droplets with time, with the distribution shifting towards larger droplet size. Figure 3 also reveals that a large number of small droplets coalesce to form bigger droplets, thus resulting in polydisperse behavior. Figure 3 illustrates that with evolution in time, the morphology of the droplets has changed and the distribution has shifted towards larger droplets.
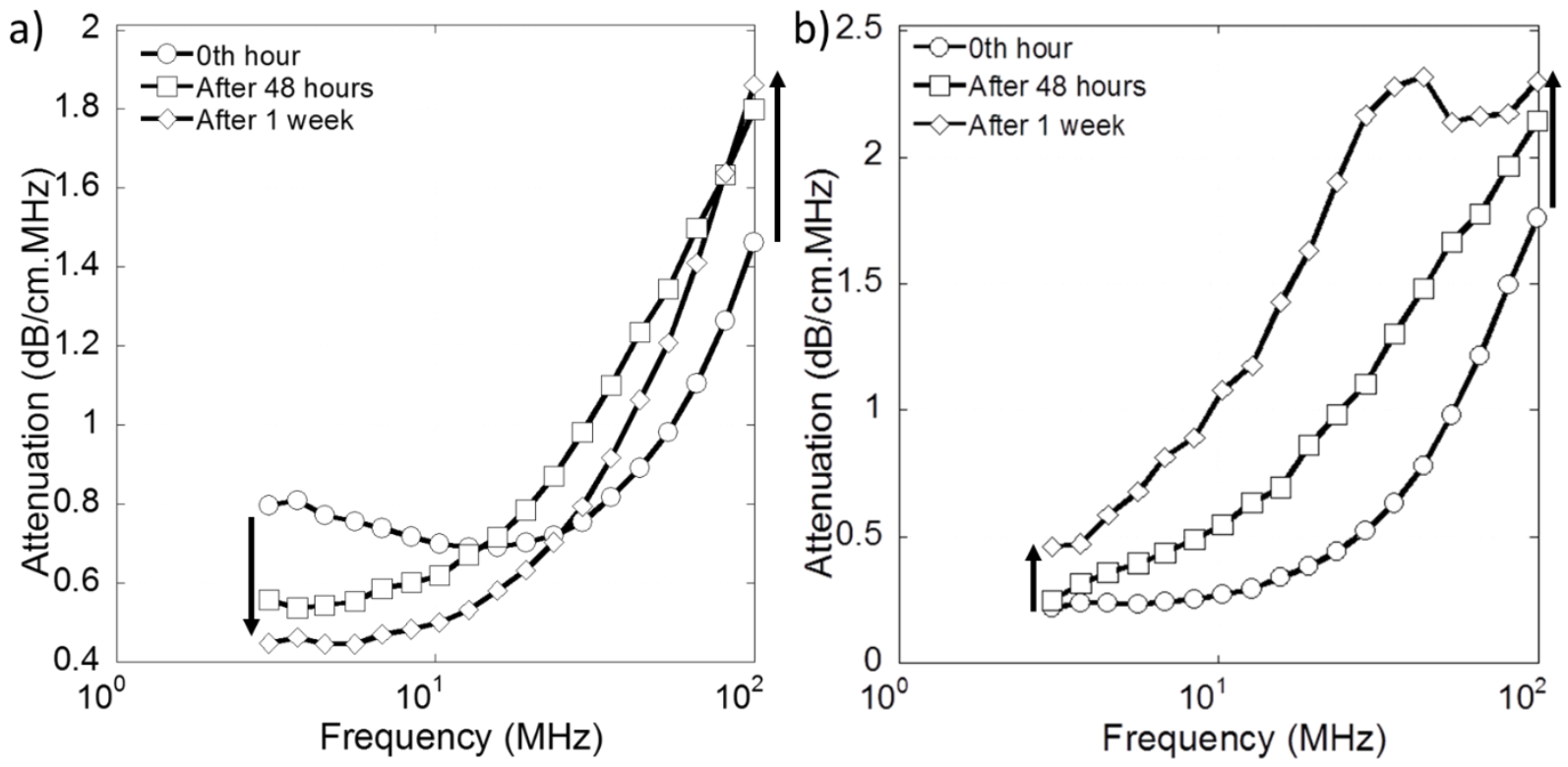
Figure 4: Attenuation spectra of a) surfactant stabilized emulsion b) solid stabilized emulsion at various time intervals using acoustic spectrometer. Arrows show the trend in the attenuation spectra immediately and after 1 week of emulsion preparation at low and high frequency.
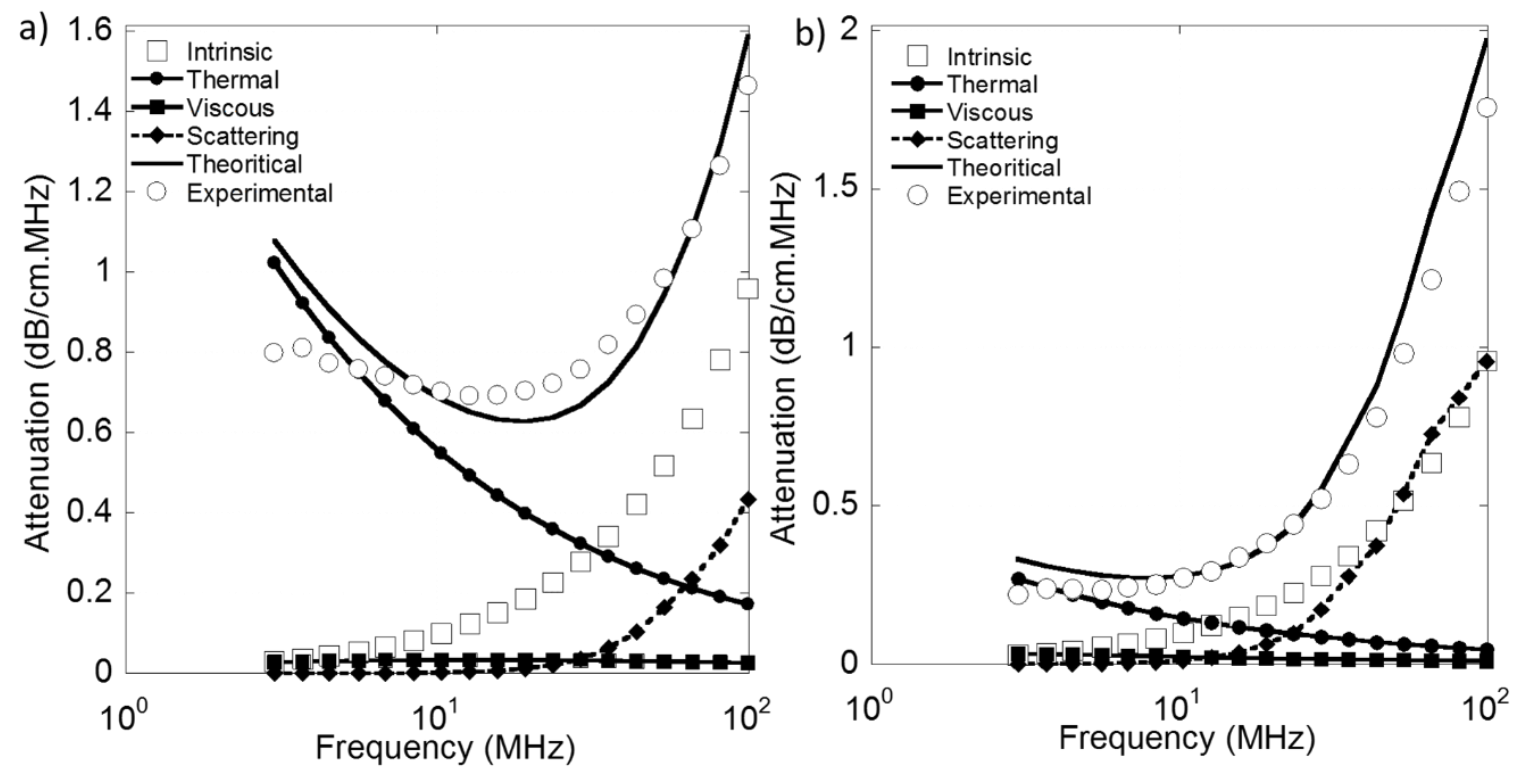

Figure 5: Experimental and theoretical attenuation spectra of water-in-oil emulsion measured immediately after sample preparation $\left(0^{\text {th }}\right.$ hour attenuation) a) Attenuation spectra of surfactant stabilized emulsion assuming thermal loss mechanism b) Attenuation spectra of solid stabilized emulsion assuming scattering loss mechanism due to the presence of large droplets

Figures 4 a) and 4 b) show the attenuation spectra of surfactant and solid stabilized emulsion respectively at three different time intervals $\left(0^{\text {th }}\right.$ hour, after 48 hours and after 1 week of emulsification). Figures 4 a) and 4 b) show the evolution of attenuation spectra over the entire frequency range (1-100 Mhz). Figure 4 a) indicates that for surfactant stabilized emulsions, the low frequency attenuation begins to decay with time. Whereas, this trend was not observed in solid stabilized emulsions (Figure $4 \mathrm{~b}$ ). The high frequency attenuation (>10 MHz) was observed to increase for both kinds of emulsions with time. Dukhin and Goetz also observed a similar 
trend in the evolution of attenuation spectra for span80 stabilized emulsions [15]. The authors observed that for span80 stabilized water-in-kerosene oil emulsion, the attenuation at lower frequency decays with time, whereas, at higher frequencies, it increases over time [15]. The measured raw attenuation is a combination of intrinsic, thermal, and scattering loss mechanisms. For this work, intrinsic loss is the attenuation caused by the continuous oil phase (decalin). As seen in Figure 5 a), for surfactant stabilized emulsion, the thermal loss mechanism contributes to the overall attenuation. Whereas, for solid stabilized emulsion, the scattering loss mechanism dominates the attenuation spectra over the entire frequency bandwidth with a decay in the attenuation due to thermal loss at higher frequencies (Figure 5 b). High frequency attenuation is caused by the scattering loss mechanism which is sensitive to a large droplet size [13]. For the two emulsion systems studied in this paper, the search routine in the DT-1202 software was modified to take into account only the thermal loss mechanism for surfactant stabilized emulsions and scattering loss for solid stabilized emulsions. It was seen that the theoretical attenuation fits the experimental data with a fitting error of $<15 \%$ for both kinds of emulsions at all the measured time intervals. The thermal loss mechanism is sensitive at lower frequencies (up to $10 \mathrm{MHz}$ ) and small droplet sizes $[13,15]$. Whereas, the scattering loss mechanism is sensitive to higher frequencies and large droplet sizes $[13,15]$. The evolution of attenuation directly indicates variation in the droplet size distribution. An increase in both the low and high frequency attenuation, as seen in Figure 4 b), indirectly indicates changes in the physical and morphological properties of the system such as droplet coalescence, polydispersity, and broad droplet size distributions. 

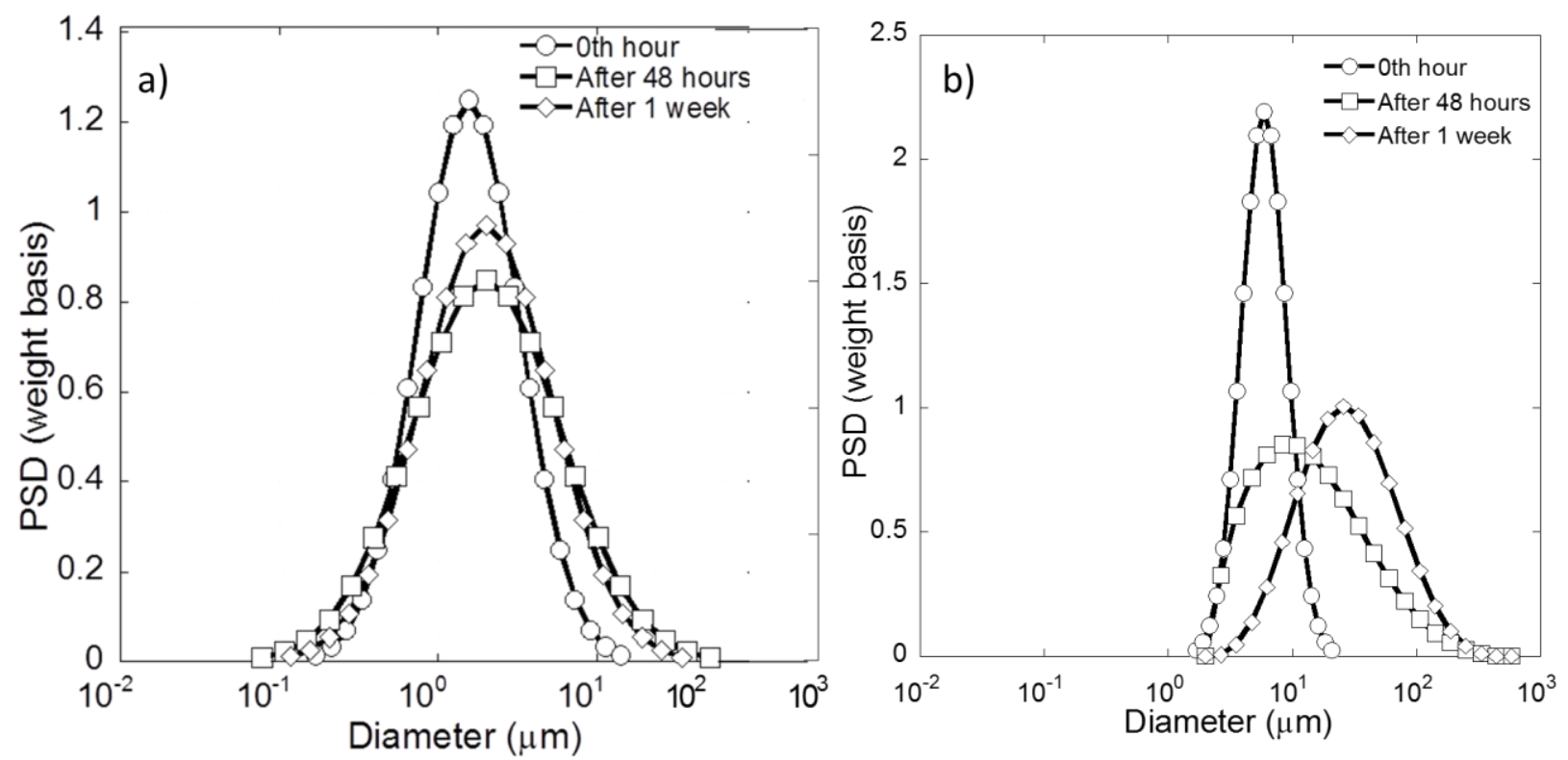

Figure 6: Drop size distributions of a) surfactant stabilized emulsion b) solid stabilized emulsion at various time intervals using acoustic spectrometer

Table 1: Average (mean) water droplet size in span80 and fumed silica stabilized emulsion measured at different time intervals after emulsion preparation using acoustic spectrometer

\begin{tabular}{|c|c|c|}
\hline Sample & $\begin{array}{c}\text { Time after emulsion } \\
\text { preparation }\end{array}$ & Mean $(\boldsymbol{\mu m})$ \\
\hline \multirow{2}{*}{$\begin{array}{c}\text { Surfactant stabilized } \\
\text { emulsion }\end{array}$} & $0^{\text {th }}$ hour & $2.0 \pm 0.32$ \\
& $48^{\text {th }}$ hour & $3.4 \pm 0.47$ \\
\hline Solid stabilized & After 1 week & $3.1 \pm 0.40$ \\
emulsion & $0^{\text {th }}$ hour & $6.4 \pm 0.18$ \\
& $48^{\text {th }}$ hour & $23.9 \pm 0.60$ \\
& After 1 week & $40.0 \pm 0.45$ \\
\hline
\end{tabular}


The acoustic spectrometer predicts the droplet size distribution based on raw attenuation loss mechanism. Droplet size distribution shown in Figures 6 a) and 6 b) are lognormal distribution that are generated as a result of automatic calculation performed by the DT 1202 software [13]. Figure 6 a) and Table 1 indicate that for the surfactant stabilized emulsion, no further increase in the mean droplet size is seen after 1 week of emulsification, and ceases after reaching $3 \mu \mathrm{m}$. This could be attributed to the fact that there is levelling of attenuation at both low and high frequencies, as seen in Figure 4 a). This behavior indicates that the emulsion is stable against de-stabilization mechanisms such as coalescence. These experimental results demonstrate that acoustic spectroscopy was successful in characterizing transient behavior exhibited by highly stable emulsions.

Table 1 and Figure $6 \mathrm{~b}$ ) indicate that droplet size evolves with time and leads to broad and polydisperse droplet size distributions for solid stabilized emulsions. Figure $4 \mathrm{~b}$ ) shows that with time, attenuation at both the low and high frequency regions increases, thereby indicating the generation of small and large micron sized, polydisperse droplets. Experimental raw attenuation and droplet size distribution of solid stabilized emulsion establishes that acoustic technique can be employed to characterize emulsions that exhibit features unique to a destabilization mechanism such as coalescence. Bottle test results shown in Figure 1, also support this observation. Images shown in Figure 3 illustrate that for solid stabilized emulsions over time, a large number of small droplets coalesce to form bigger droplets. This behavior results in a shift in the distribution toward larger droplets.

Emulsion destabilization mechanisms in water-in-oil emulsion mainly arises due to coalescence, Ostwald ripening, flocculation, and sedimentation. Evolution of droplet size or irreversible coarsening of emulsion occurs largely due to coalescence or Ostwald ripening. 
Ostwald ripening is primarily driven by a difference in laplace pressure between droplets of different sizes, where the dispersed phase is transferred from smaller to larger droplets $[16,17]$. Whereas, coalescence occurs due to rupture of interfacial film between droplets. Mostly, emulsion destabilization studies result from interplay between Ostwald ripening and coalescence, where only one of the two mechanism is dominant at a given time [16]. Experiments, as well as theoretical models, have divulged that Ostwald ripening generates narrow size distribution, whereas coalescence results in growth of large droplets that rapidly separate out as an oil or aqueous phase layer at the top or bottom of the sample [16-24]. Binks and others have observed that emulsions are stable against coalescence when the droplets are completely covered by a hexagonal closed packed monolayer of particles [19, 25-27]. Several studies have shown that the emulsion droplet size and ratio of the amount of particles available to that required for complete coverage, is a function of particle concentration $[4,25,28,29]$. Excess particles in the continuous phase were found to result in smaller droplet size emulsions, remain suspended in the continuous phase causing increase in the viscosity, and produce emulsions stable against coalescence by forming a network of particles entrapping the water droplets [9, 29]. Depending on the amount of particles available and adsorbed on the droplet interface, the droplets that have insufficient surface coverage will continue to coalesce until a dense solid interfacial film is formed that inhibits coalescence and kinetically stabilizes the emulsions $[9,16,26,30]$. This results in a decrease in the total surface area until there is a sufficient amount of solid particles available to stabilize the droplets $[29,31]$.

Previous work by Levine, Binks and many others suggest that knowing the oil concentration and the interfacial area per unit mass of silica particles, the amount of solid particles required for maximum packing can be calculated $[4,25,28,29]$. For this work, the 
interfacial area per unit mass of silica particles was calculated using the relationship predicted by Wiley and Arditty et al. [26, 27]. For the purpose of this work, we calculated that the amount of silica needed to achieve dense monolayer coverage was $4.08 \mathrm{~g}$. The amount of silica available (the mass of silica particles in the emulsion was $2 \mathrm{~g}$ ) was found to be less than the amount required to achieve monolayer coverage $(4.08 \mathrm{~g})$. In addition to this, the solid particles used in this study are highly hydrophobic which increases the tendency of these particles to remain in the oil phase and makes them less likely to be adsorbed at the interface. This suggests that lack of solid particles at the interface resulted in an increase in mean droplet size and hence, coalescence is the dominant destabilization mechanism.

\section{Conclusions and Future Work}

In this paper, the drop size distribution, and transient stability of water-in-oil emulsion were stabilized using a non-ionic surfactant (Span80) and hydrophobic fumed silica (aerosil R972) was measured using acoustic spectroscopy. Differences in terms of the evolution of drop size distribution was observed at various time intervals. Increase in the attenuation at low and high frequencies for solid stabilized emulsions illustrate that droplet coalescence occurred, thereby leading to polydisperse morphologies and large droplet size emulsions. Optical microscopy results validate the droplet size distribution results obtained by acoustic spectroscopy. The acoustic spectroscopy technique can be used to characterize the transient behavior of emulsions as it captures both morphological and physical changes in the emulsions through raw experimental attenuation.

Further experiments and evaluation of the droplet size distribution and transient stability for mixed surfactant and solid stabilized emulsions as a function of water concentration and emulsifier concentration would provide more insight into emulsion stabilization mechanism and 
interfacial properties. To better understand the emulsion stabilization and destabilization mechanisms using acoustic spectroscopy, further work will be performed on specific interfacial and rheological interactions between surfactant molecules and solid particles.

\section{Acknowledgements}

The authors thank the Department of Energy (DOE/EPSCOR) program for funding this work (Grant DE-SC0004600). The authors thank Dr. Victor Lifton (Evonik Inc.) for donating samples for this work. The authors thank Dr. Andrei Dukhin and Sean Parlia (Dispersion Technology Inc.) for consultation and their help in analyzing our data. The authors thank Ashwin Yegya Raman (Oklahoma State University) for his help in the laboratory. The authors thank Dr.

Peter Clark (Oklahoma State University) and Ruben Nieblas (McCrone Microscope \& Accessories) for their help with the optical microscope. The authors give special thanks to Mrs. Eileen Nelson for editing the manuscript.

\section{References}

[1] A. Drelich, F. Gomez, D. Clausse, and I. Pezron, "Evolution of water-in-oil emulsions stabilized with solid particles," Colloids and Surfaces A: Physicochemical and Engineering Aspects, vol. 365, pp. 171-177, 2010.

[2] S. U. Pickering, "Pickering Emulsions," Journal of Chemical Society, vol. 91, 1907.

[3] B. P. Binks, "Particles as surfactants similarities and differences," Colloid and Interface Science, vol. 7, pp. 21-41, 2002.

[4] S. Levine, B. D. Bowen, and S. J. Partridge, "Stabilization of emulsions by fine particles I. Partitioning of particles between continuous phase and oil/water interface," Colloids and Surfaces, vol. 38, pp. 325-343, // 1989.

[5] B. P. Binks and J. H. Clint, "Solid Wettability from Surface Energy Components: Relevance to Pickering Emulsions," Langmuir, vol. 18, pp. 1270-1273, 2002.

[6] F. Leal-Calderon and V. Schmitt, "Solid-stabilized emulsions," Current Opinion in Colloid \& Interface Science, vol. 13, pp. 217-227, 2008.

[7] B. P. Binks and S. O. Lumsdon, "Influence of Particle Wettability on the Type and Stability of Surfactant-Free Emulsions," Langmuir, vol. 16, pp. 8622-8631, 2000.

[8] P. Finkle, H. D. Draper, and J. H. Hildebrand, "The Theory of Emulsification I," Journal of the American Chemical Society, vol. 45, pp. 2780-2788, 1923/12/01 1923.

[9] J. Frelichowska, M.-A. Bolzinger, and Y. Chevalier, "Effects of solid particle content on properties of o/w Pickering emulsions," Journal of Colloid and Interface Science, vol. 351, pp. 348-356, 11/15/2010. 
[10] A. S. Dukhin and P. J. Goetz, "Acoustic and electroacoustic spectroscopy," Langmuir, vol. 12, pp. 4336-4344, 1996.

[11] A. S. Dukhin, Ultrasound for Characterizing Colloids Particle Sizing, Zeta Potential Rheology: Elsevier, 2002.

[12] M. K. N. M. Chatterjee, B. Siladitya, and D. Ganguli, "Role of organic solvents and surface-active agents in the sol-emulsion-gel synthesis of spherical alumina powders," Journal of Materials Research, vol. 15, pp. 176-185, Jan., 20001999.

[13] A. S. Dukhin and P. J. Goetz, Characterization of Liquids, Nano- and Microparticulates, and Porous Bodies using Ultrasound 2ed.: Elsevier, 2010.

[14] A. S. Dukhin and P. J. Goetz, "Acoustic and electroacoustic spectroscopy for characterizing concentrated dispersions and emulsions," Advances in Colloid and Interface Science, vol. 92, pp. 73-132, 20012001.

[15] A. Dukhin and P. Goetz, "Evolution of water-in-oil emulsion controlled by droplet-bulk ion exchange: acoustic, electroacoustic, conductivity and image analysis," Colloids and Surfaces A: Physicochemical and Engineering Aspects, vol. 253, pp. 51-64, 2005.

[16] F. Leal-Calderon, V. Schmitt, and J. Bibette, Emulsion Science - Basic Principles, Second ed.: Springer, 2007.

[17] A. S. Kabalnov, A. V. Pertzov, and E. D. Shchukin, "Ostwald ripening in emulsions," Journal of Colloid and Interface Science, vol. 118, pp. 590-597, 1987/08/01 1987.

[18] A. S. Kabalnov, K. N. Makarov, A. V. Pertzov, and E. D. Shchukin, "Ostwald ripening in emulsions: 2. Ostwald ripening in hydrocarbon emulsions: Experimental verification of equation for absolute rates," Journal of Colloid and Interface Science, vol. 138, pp. 98$104,8 / / 1990$.

[19] T. S. Horozov and B. P. Binks, "Particle-Stabilized Emulsions: A Bilayer or a Bridging Monolayer?," Angewandte Chemie International Edition, vol. 45, pp. 773-776, 2006.

[20] J. Bibette, D. C. Morse, T. A. Witten, and D. A. Weitz, "Stability criteria for emulsions," Physical Review Letters, vol. 69, pp. 2439-2442, 10/19/ 1992.

[21] D. J. Durian, D. A. Weitz, and D. J. Pine, "Scaling behavior in shaving cream," Physical Review A, vol. 44, pp. R7902-R7905, 12/01/ 1991.

[22] A. Hasmy, R. Paredes, O. Sonneville-Aubrun, B. Cabane, and R. Botet, "Dynamical Transition in a Model for Dry Foams," Physical Review Letters, vol. 82, pp. 3368-3371, 04/19/ 1999.

[23] I. M. Lifshitz and V. V. Slyozov, "The kinetics of precipitation from supersaturated solid solutions," Journal of Physics and Chemistry of Solids, vol. 19, pp. 35-50, 1961/04/01 1961.

[24] C. Wagner, "Theory for the Coarsening of Solid Precipitates Caused by Ostwald Ripening," Z. Elektrochem, vol. 65, p. 581, 1961.

[25] V. B. Menon and D. T. Wasan, "Characterization of Oil-Water Interfaces Containing Finely Divided Solids with Applications to the Coalescence of Water-in-Oil Emulsion: A review," Colloid and Surfaces, vol. 29, pp. 7-27, 1988.

[26] R. M. Wiley, "Limited coalescence of oil droplets in coarse oil-in-water emulsions," Journal of Colloid Science, vol. 9, pp. 427-437, 10// 1954.

[27] S. Arditty, C. P. Whitby, B. P. Binks, V. Schmitt, and F. Leal-Calderon, "Some general features of limited coalescence in solid-stabilized emulsions," Eur. Phys. J. E, vol. 11, pp. 273-281, 2003. 
[28] B. P. Binks and S. O. Lumsdon, "Pickering Emulsions Stabilized by Monodispersed Latex Particles: Effects of Particle Size," Langmuir, vol. 17, pp. 4540-4547, 2001.

[29] Y. Yan and J. H. Masliyah, "Solids-stabilized oil-in-water emulsions: Scavenging of emulsion droplets by fresh oil addition," Colloids and Surfaces A: Physicochemical and Engineering Aspects, vol. 75, pp. 123-132, 8/10/ 1993.

[30] M. Destribats, S. Ravaine, V. Heroguez, F. Leal-Calderon, and V. Schmitt, "Outstanding Stability of Poorly-protected Pickering Emulsions," in Trends in Colloid and Interface Science XXIII. vol. 137, ed: Springer Berlin Heidelberg, 2010, pp. 13-18.

[31] D. E. Tambe and M. M. Sharma, "Factors Controlling the Stability of Colloid-Stabilized Emulsions: I. An Experimental Investigation," Journal of Colloid and Interface Science, vol. 157, pp. 244-253, 4// 1993. 


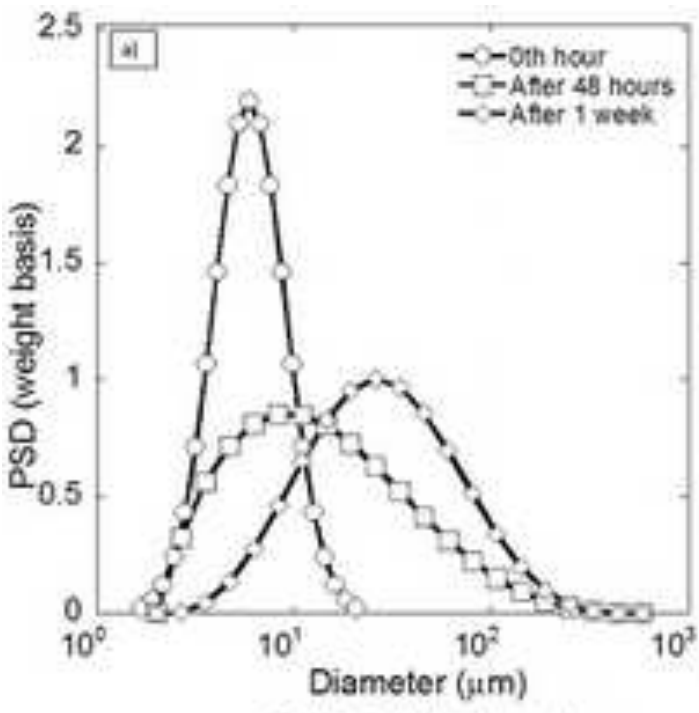

Solid stabilized emulsion
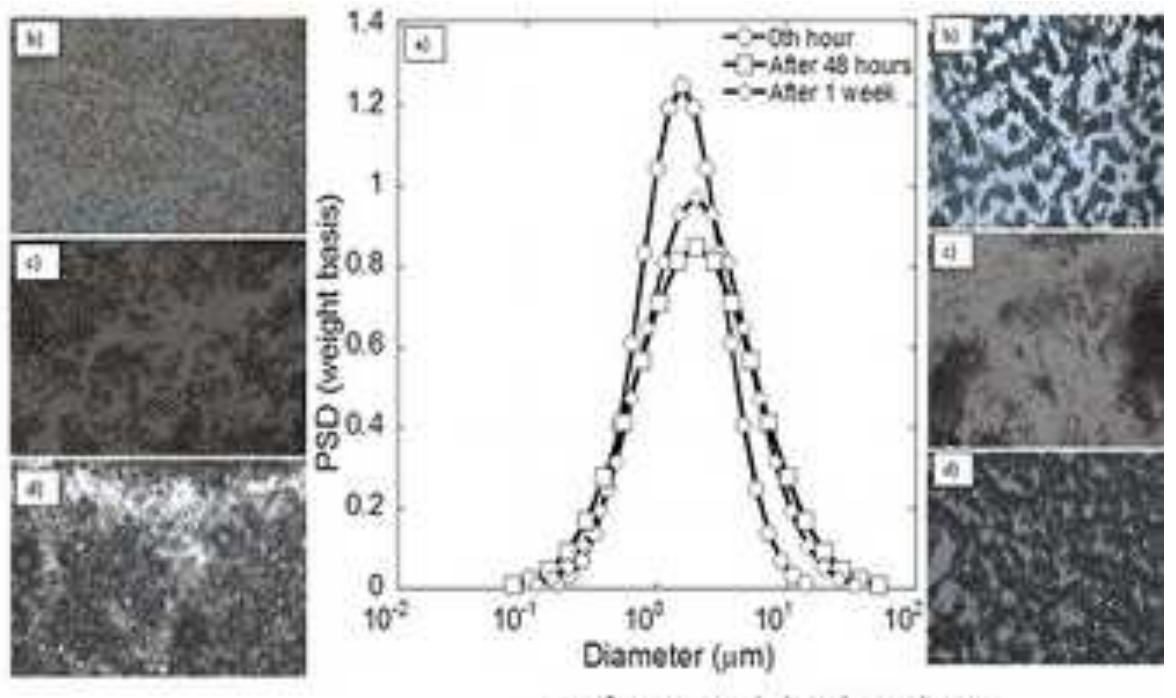

Surfactant stabilized emulsion 